\title{
Research Paper \\ Predicting Resilience Based on Dark Triad Personality and Psychological Wellbeing in Athletes Students
}

\author{
Farzin Bagheri Sheykhangafshe ${ }^{1}\left(\mathbb{C},{ }^{*}\right.$ Abbas Abolghasemi ${ }^{2} \odot$, Seyyed Mousa Kafi Masouleh ${ }^{2}$
}

1. Department of Psychology, Faculty of Humanities, Tarbiat Modares University, Tehran, Iran

2. Department of Psychology, Faculty of Literature and Humanity Sciences, University of Guilan, Rasht, Iran.

\begin{tabular}{|c|c|}
\hline $\begin{array}{l}\text { Use your device to scan } \\
\text { and read the article online }\end{array}$ & ykhangafshe F, Abolghasemi A, Kafi Masouleh SM. [Predicting Resilience Based on Dark Triad Person- \\
\hline 口ring & ality and Psychological Wellbeing in Athletes Students (Persian)]. Journal of Arak University of Medical Sciences (JAMS). 2021; \\
\hline $4+4+4$ & 24(2):230-245. https://doi.org/10.32598/JAMS.24.2.6151.1 \\
\hline aly룰 & dol'https://doi.org/10.32598/JAMS.24.2.6151.1 \\
\hline
\end{tabular}

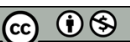

Article Info:

Received: 15 Apr 2020

Accepted: 06 Jul 2021

Available Online: 01 Jun 2021

Key words:

Resilience, Dark triad personality, Psychological wellbeing, Students, Athlete

\section{A B STRACT}

Background and Aim The concept of resilience has been recently extensively considered concerning sports studies; accordingly, in most qualitative studies, it has been used to explore the attitudes of coaches, athletes, and sports psychologists. In this regard, the present study aimed to determine resilience in athletic students based on dark triad personality and psychological wellbeing.

Methods \& Materials This was a descriptive and correlational study. The population of the study consisted of all male students of Guilan University of Physical Education in the first semester of the academic year 2019-2020. A sample of 200 individuals was selected by convenience sampling method; then, the required data were collected using Sheard et al.'s (2009) Resilience Scale for Adults, Jonsson and Webster's Dark Triad Personality Questionnaire, and Ryff's 18-item Psychological Wellbeing Scale (1989). The obtained data were analyzed by SPSS using Pearson correlation coefficient and multiple regression analysis. Ethical Considerations This study was approved by Research Ethics Committee of Guilan University of Medical Sciences (Code: IR.GUMS.REC.1398.121).

Results The present research results suggested a positive and significant correlation between the resilience of the examined athletic students and narcissism ( $r=0.495, P<0.001)$, self-acceptance $(r=0.598$, $P<0.001)$, purposefulness in life $(r=0.556, P<0.001)$, personal growth $(r=0.648, P<0.001)$, the mastery of the environment $(r=0.645, P<0.001)$, positive correlation with others $(r=0.569, P<0.001)$, and independence $(r=0.381, P<0.001)$. Moreover, there was a significant negative correlation between antisocial behavior $(r=-0.557, P<0.001)$ and Machiavelli's aspect $(r=-0.604, P<0.001)$, and the explored students' resilience. Additionally, multiple regression analysis data revealed that dark triad personality and psychological wellbeing predict $62 \%$ of resilience in the study subjects.

Conclusion The current investigation results signified a significant correlation between dark triad personality and psychological wellbeing, and resilience in athletic students. Accordingly, educators and teachers need to pay more attention to the role of psychological wellbeing and different dimensions of athletes' personalities for promoting the health and performance of students.

* Corresponding Author:

Abbas Abolghasemi, PhD.

Address: Department of Psychology, Faculty of Literature and Humanity Sciences, University of Guilan, Rasht, Iran.

Tel: +98 (13) 66902759

E-mail: abolghasemi1344@guilan.ac.ir 


\section{Extended Abstract}

\section{Introduction}

I

$\mathrm{n}$ addition to sports activities, athletes pursue university education related to their field of sport, i.e., known in the literature of sports psychology as the achievement of a dual goal [1]. Professional athletes have different psychological characteristics, compared to others; these characteristics can help them perform at their best in competition. Resilience is defined as a set of attitudes and behaviors that enable the athlete to overcome any pressure by effort and to concentrate on moving towards the goals [12]. Dark personality traits consist of Machiavellian traits, narcissism, and antisocial behavior. These traits are in a conceptual cluster due to having common dimensions, such as undesirable social nature, hypocrisy, the lack of empathy, aggression, self-centeredness, and deception [17]. Psychological wellbeing, along with reducing negative emotions and anxiety, helps the athlete to create a lively and desirable training environment and take a firm step towards increasing performance [28].

\section{Materials and Methods}

The present study was of a descriptive and correlational design. The study population included all physical education students of Guilan University in 2019. Of them, 200 student athletes were included in the study by convenience sampling method. The inclusion criteria of the study were healthy physical conditions, male gender, the age range of 18-40 years, and a history of sports activity in the last 2 years. The exclusion criteria included not belonging to the research community, a probationary history, presenting acute psychological problems, being able to answer the questions, and providing incomplete questionnaires. The following tools were employed in this study to collect the necessary data:

Resilience Scale for Athletes: This is a self-assessment tool, consisting of 14 items and 3 factors that measure thoughts and cognitions related to confidence, stability, and control. Each item is scored on a five-point Likert-type scale [33].

The Dark Triad Personality Test: This scale was developed by Jonason and Webster [33] and includes 12 items and 3 factors: Machiavellianism, narcissism, and antisocial behavior. Besides, the tool measures these factors with 12 questions, answered on a 9-point Likert-type scale.

Psychological Wellbeing Scale: This scale was designed by Ryff in 1989 [23]. The 18-item form of this scale in- cludes 6 factors of independence, mastery of the environment, personal growth, positive communication with others, purposefulness in life, and self-acceptance, i.e., graded on a 6-point Likert-type scale [24].

\section{Results}

Table 1 presents the descriptive indicators of research variables. Furthermore, the results of the Shapiro-Wilk test are reported to check the normality of the distribution of research variables (Table 1). The results of the Shapiro-Wilk test were not significant for all variables; thus, the distribution of the study variables was normal.

The results of the Pearson correlation coefficient indicated that Machiavellianism and antisocial behavior were significantly and negatively correlated with resilience $(\mathrm{P}<0.01)$. In contrast, narcissism, independence, mastery of the environment, personal growth, positive communication with others, purposefulness in life, and self-acceptance had a significant positive correlation with resilience $(\mathrm{P}<0.01)$.

Table 2 presents the results of examining the role of dark personality traits and psychological wellbeing in predicting resilience among the explored student athletes. The relevant results revealed that the correlation coefficient of predictor variables with the resilience of student-athletes equaled 0.78 ; these 9 variables could significantly predict $62 \%$ of changes in resilience $(\mathrm{P}<0.001)$. Moreover, antisocial behavior and Machiavellianism were able to negatively predict resilience in the study subjects. In contrast, self-acceptance, purposefulness in life, narcissism, personal growth, mastery of the environment, positive communication with others, and independence could positively and directly predict resilience among the study participants.

\section{Discussion and Conclusion}

The present study data indicated a significant negative correlation between Machiavellian traits, antisocial behavior, and resilience. Furthermore, a significant positive correlation was found between narcissism and mental strength in the explored student athletes. In other words, individuals who achieved high scores in Machiavellianism and antisocial behavior only seek to satisfy their desires and do not pay attention to the rights of others, which in turn, causes instability in their behavior. In contrast, athletes with welladjusted levels of narcissism strive to achieve their goals and act on their talents and abilities [15-18]. In this regard, Unoe et al. [21] examined the correlation between competitive sports and dark personality traits; they concluded that the effects of gender, type of sports activity, and level of 
Table 1. Descriptive indicators and results of examining the normal distribution of research variables

\begin{tabular}{cccc}
\hline Characteristic & Mean \pm SD & Shapiro Wilk's Test & P \\
\hline Resilience & $52.97 \pm 7097$ & 0.902 & 0.95 \\
\hline Machiavellianism & $14.22 \pm 6071$ & 0.914 & 0.142 \\
\hline Antisocial behavior & $15.77 \pm 8009$ & 0.921 & 0.257 \\
\hline Narcissism & $16.68 \pm 6061$ & 0.892 & 0.59 \\
\hline Independence & $10.39 \pm 3021$ & 0.914 & 0.218 \\
\hline Mastery of the environment & $10.15 \pm 2039$ & 0.916 & 0.187 \\
\hline Self-Growth & $11.66 \pm 3018$ & 0.875 & 0.55 \\
\hline Positive communication with others & $10.21 \pm 2058$ & 0.893 & 0.62 \\
\hline Purposefulness in life & $41.41 \pm 209$ & 0.907 & 0.169 \\
\hline Self-Acceptance & $9.47 \pm 3002$ & 0.886 & 0.74 \\
\hline
\end{tabular}

competition in which athletes are involved largely affect the total score of dark personality traits.

Additionally, the collected findings revealed a significant positive correlation between psychological wellbeing and resilience in the study participants. Athletes with high resilience remain more stable during professional sports, have confidence in their abilities, and strive to achieve their goals with concentration and perseverance [25]. They also can control their level of anxiety in stressful situations, like sports events, and present their best performance compared to other competitors [29]. In other words, all of these characteristics are prominent features of psychological wellbeing, such as purposefulness in life, personal growth, mastery of the environment, and self-acceptance. In this regard, Mikogulari and Ekmexi [26] examined the training of psychological skills in increasing resilience; they concluded that athletes with high levels of psychological wellbeing have better resilience during competitions.

Table 2. Multiple regression analysis data for dark personality traits and psychological wellbeing

\begin{tabular}{cccccc}
\hline Characteristic & B & SE & $\boldsymbol{\beta}$ & T & P \\
\hline Fixed value & 35.50 & 2.11 & - & 16.78 & 0.001 \\
\hline Machiavellianism & -0.138 & 0.052 & -0.116 & -2.65 & 0.008 \\
\hline Antisocial behavior & -0.124 & 0.034 & -0.126 & -3.59 & 0.001 \\
\hline Narcissism & 0.139 & 0.037 & 0.117 & 3.80 & 0.001 \\
\hline Independence & 0.147 & 0.071 & 0.059 & 2.06 & 0.040. \\
\hline $\begin{array}{c}\text { Mastery of the environment } \\
\quad \text { Self-Growth }\end{array} \quad 0.282$ & 0.240 & 0.128 & 0.085 & 2.20 & 0.028 \\
\hline $\begin{array}{c}\text { Positive communication } \\
\text { with others }\end{array}$ & 0.211 & 0.096 & 0.096 & 2.49 & 0.013. \\
\hline $\begin{array}{c}\text { Purposefulness in life } \\
\text { Self-Acceptance }\end{array}$ & 0.459 & 0.098 & 0.068 & 2.14 & 0.033 \\
\hline
\end{tabular}

$\mathrm{R}=0.78 ; \mathrm{R}^{2}=0.62 ; \mathrm{F}=38.55 ; \mathrm{P}<0.001$.

Journal of 


\section{Ethical Considerations}

\section{Compliance with ethical guidelines}

This study was approved by Research Ethics Committee of Guilan University of Medical Sciences (Code: IR.GUMS.REC.1398.121). Research questionnaires were reviewed by the security and education management of Guilan School of Physical Education.

\section{Funding}

This research did not receive any grant from funding agencies in the public, commercial, or non-profit sectors.

\section{Authors' contributions}

All authors participated in the preparation of this article and met the standard writing criteria based on the recommendations of the International Committee of Medical Journal Publishers (ICMJE).

\section{Conflicts of interest}

The authors declared no conflicts of interest. 
This Page Intentionally Left Blank 


\title{
ييشبينى استحكام روانى بر اساس ركَهاى ثاريك شخصيث و بهزيستى روانشناختى در دانشجويان ورزشيثر اسشار
}

\author{
فرزين باقرى شيخانكفشه' هـ، "عباس ابوالقاسمى' مه سيد موسى كافى ماسوله' \\ ا. ا.تروه روانشناسى، دانشكده علوم انسانى، دانشكاه تربيت مدرس، تهران، ايران.

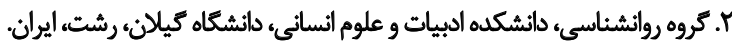

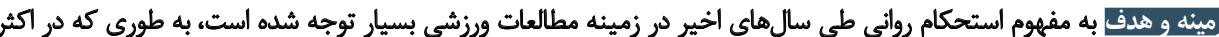

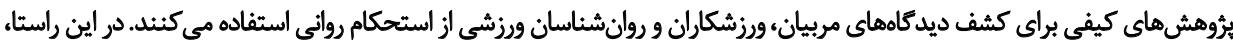

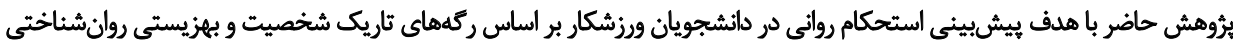

$$
\text { انجام شد. }
$$

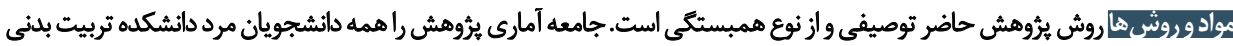

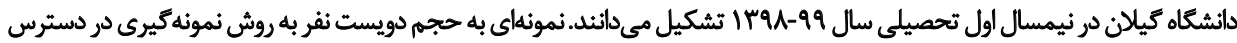

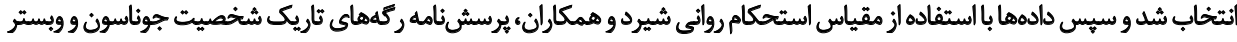

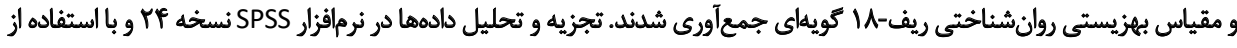

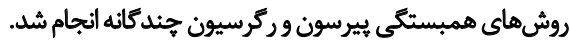

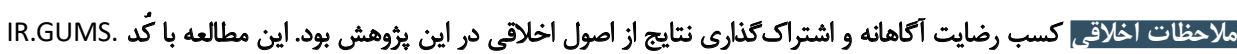

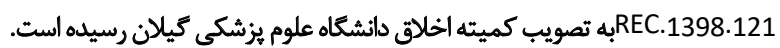

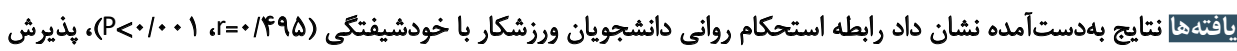

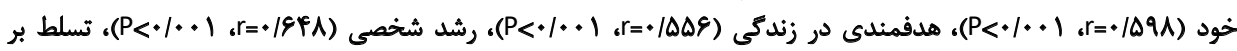

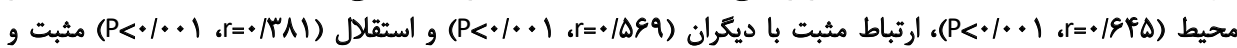

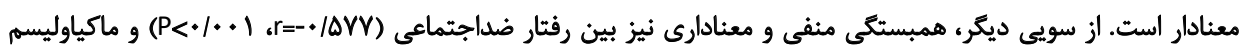

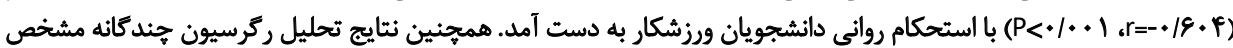

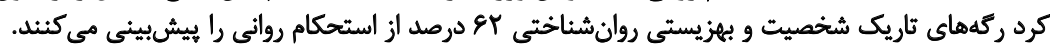

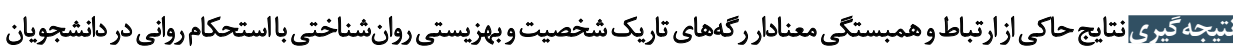

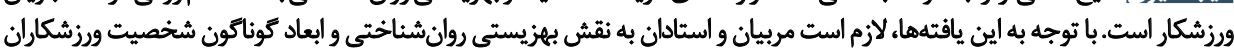

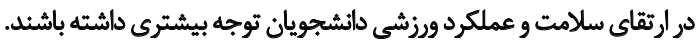

اطلاعات مقاله:

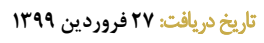

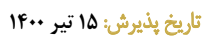

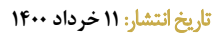

كليدوأزهها: استحكام روانى، ركههاى تاريك شخصيت، بهزيستي روانشناختي، دانشجويان، ورزشكار 
نمايش بكذارد [ [1]] در اين راستا، يكى از مهمترين مؤلفههايي

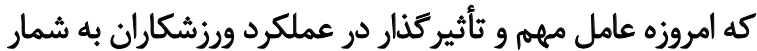

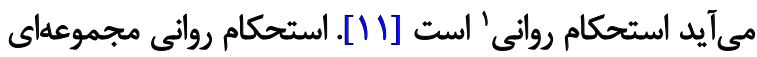

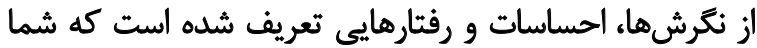

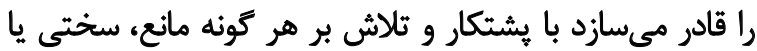

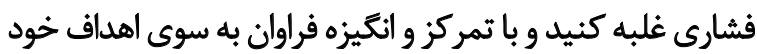

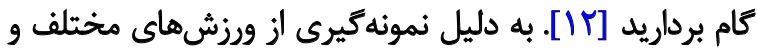

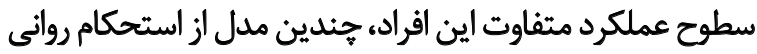

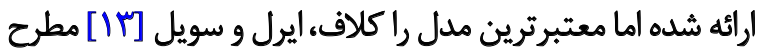

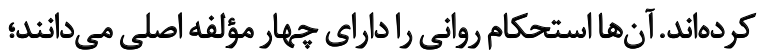

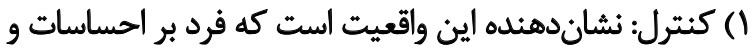

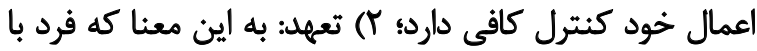

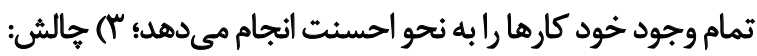

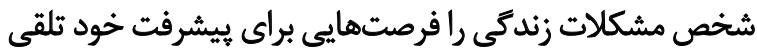

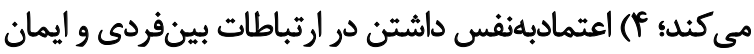

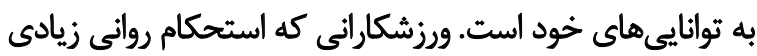

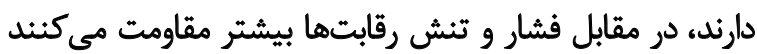

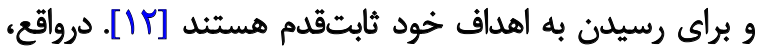

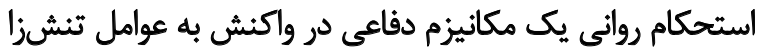

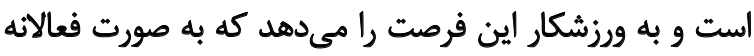

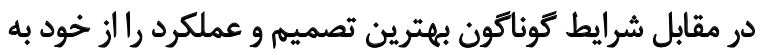

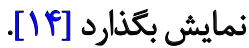

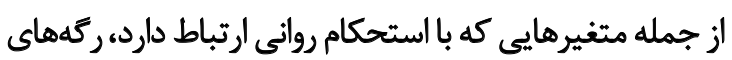

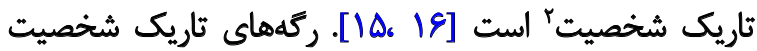

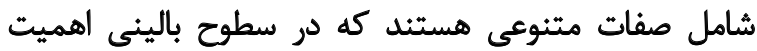

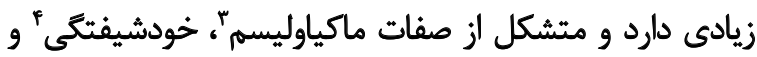

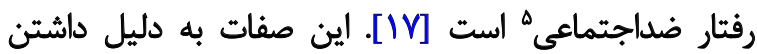

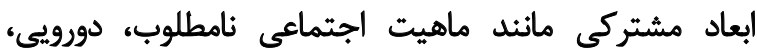

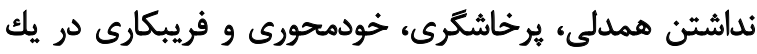

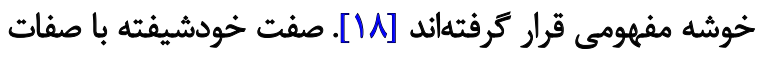

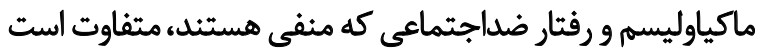

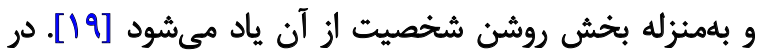

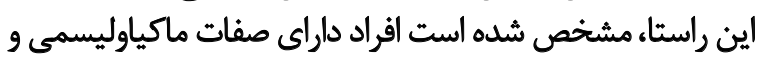

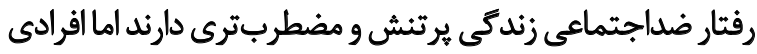

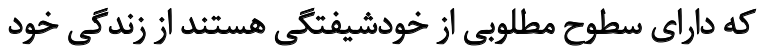

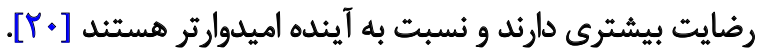

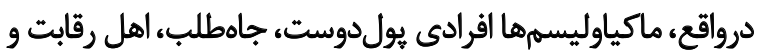

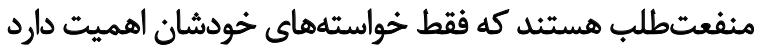

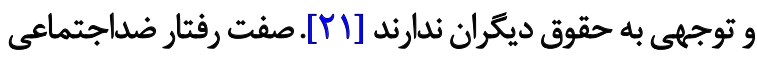

1. Mental Toughness

2. Dark triad personality

3. Machiavelli's

4. Narcissism

5. Psychopathy

ورزشكاران حرفهاى در كنار فعاليتهاى ورزشى، تحصيلات

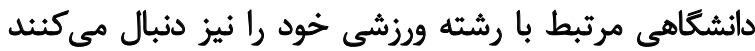

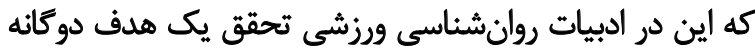

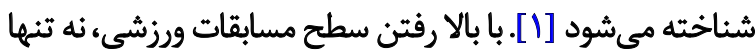

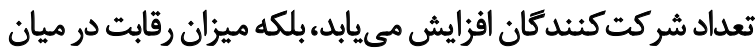

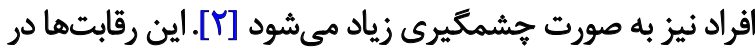

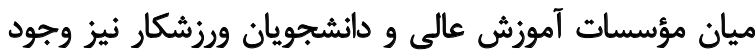

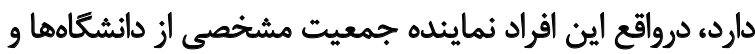

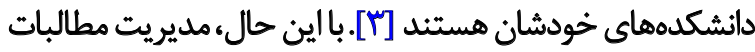

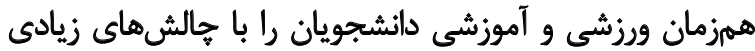

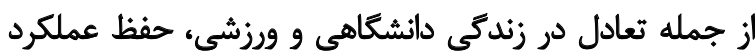

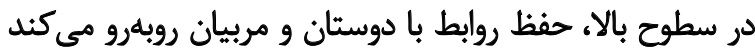

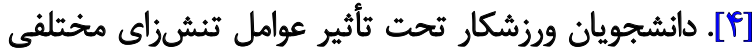

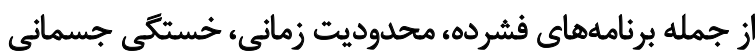

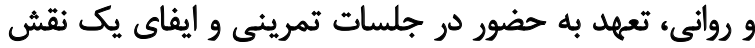

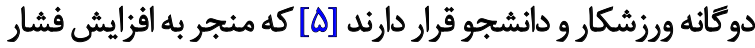

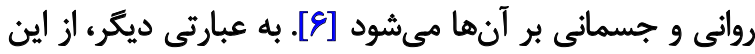

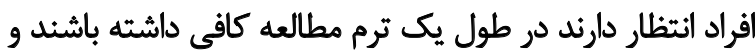

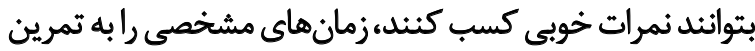

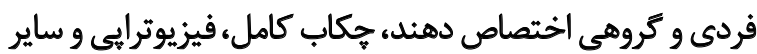

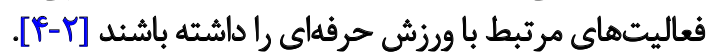

علاوه بر اين، بسيارى از اين ورزشكاران به علت فشار تمرينات

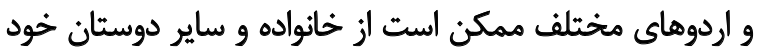

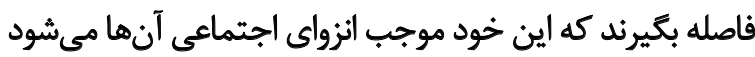

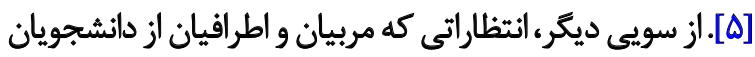

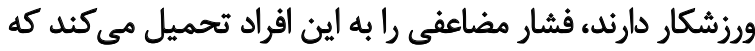

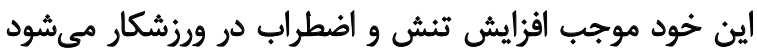

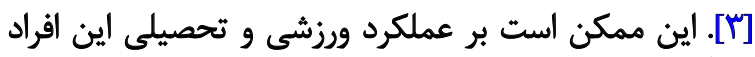

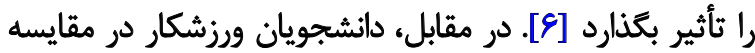

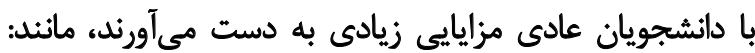

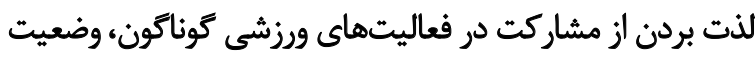

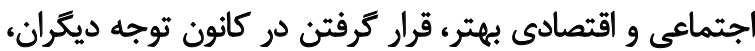

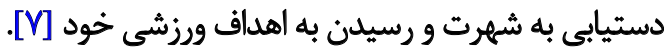

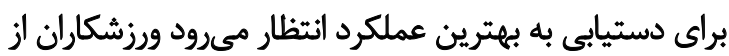

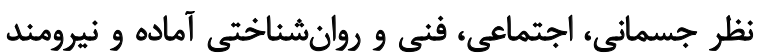

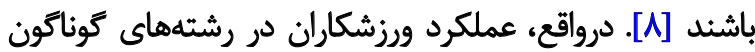

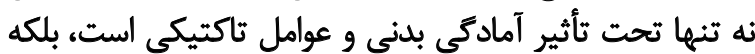

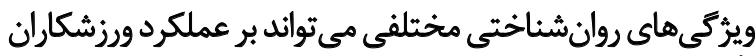

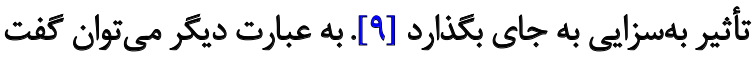

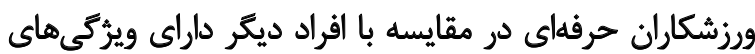

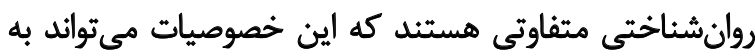

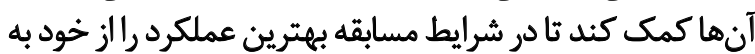


كوناكون بر عملكرد ورزشكاران را ضرورى ميدانند [عائ].

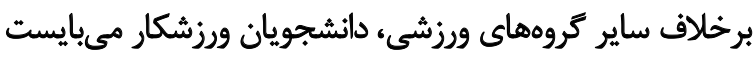

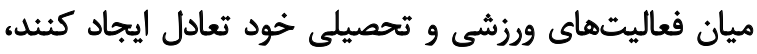

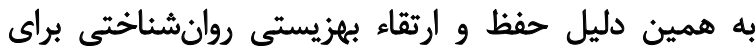

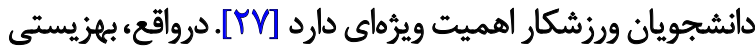

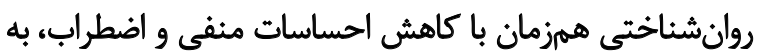

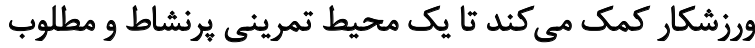

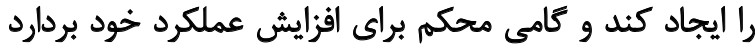

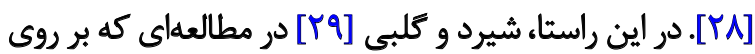

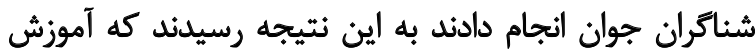

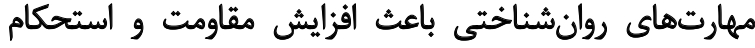

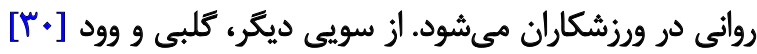

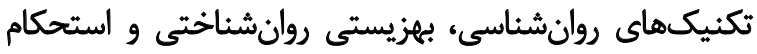

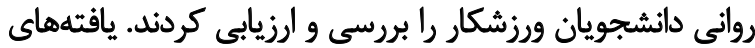

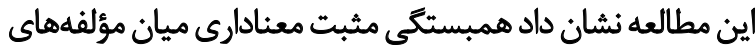

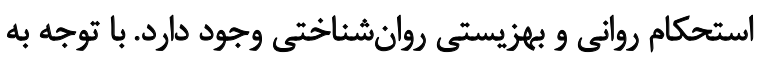

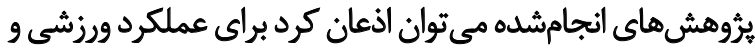

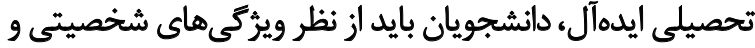

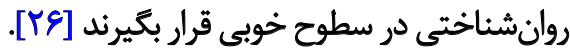

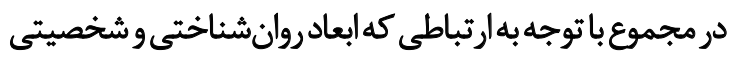

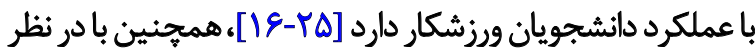

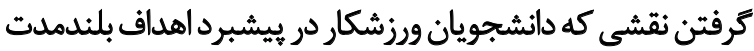

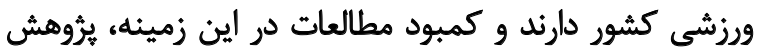

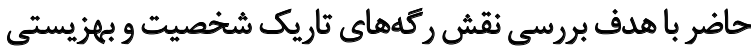
روانشناختى در بيشيشينى استحكام روانى دانشجويان ورزشكار

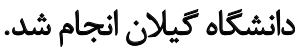

\section{مواد و روشها}

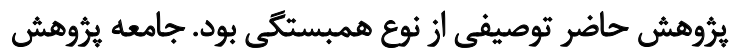

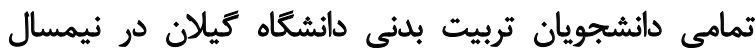

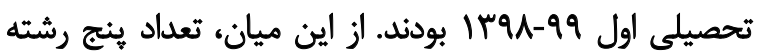

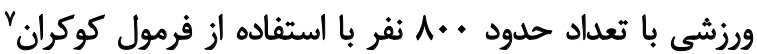

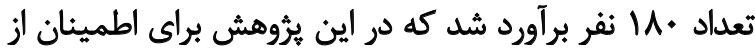

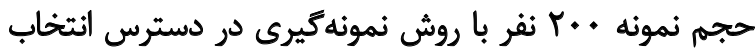

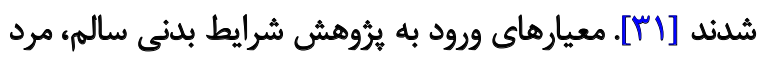

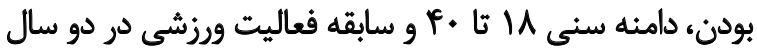

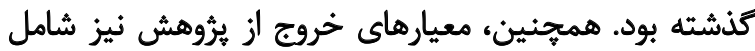

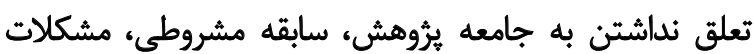

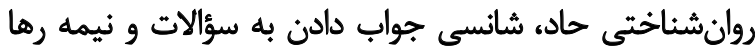
كردن يرسشنامهنها است.

نحوه اجراى بروهش به اين صورت بود كه در ابتدا اقدامات لازم براى دريافت كد اخلاق از دانشًاه علوم بز بزشكى كيلان
نيز شامل مجموعه ويرّكى هاى منفى و هنجارشكن است كه از

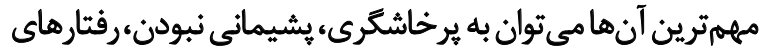

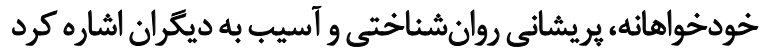

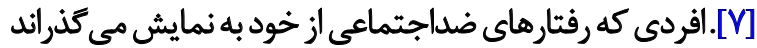

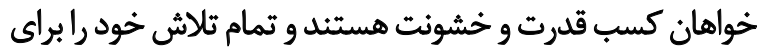

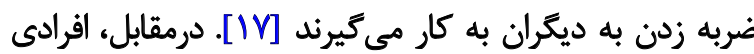

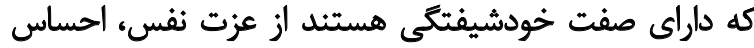

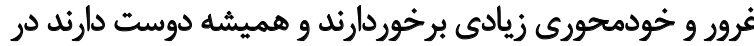

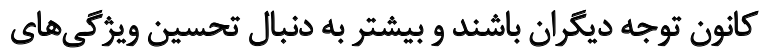

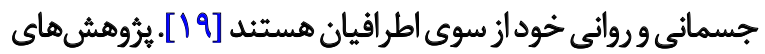

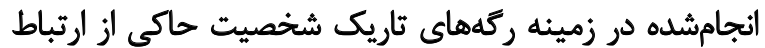

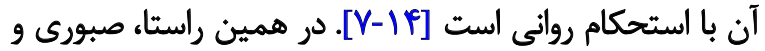

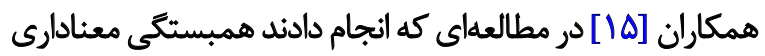

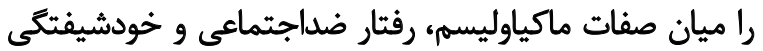

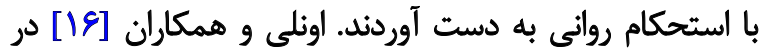

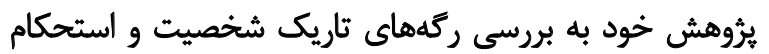

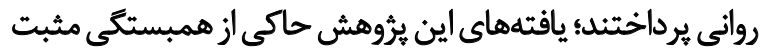

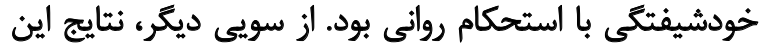

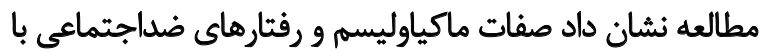

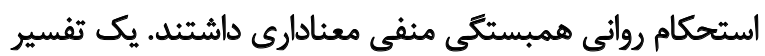

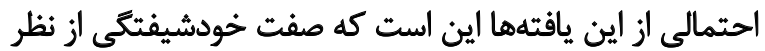

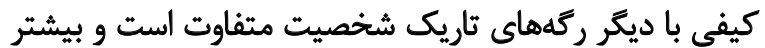

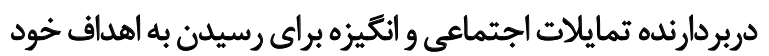

$$
\text { در زندكى است [1ه-10]. }
$$

در حالى كه بسياى از بروهشكران در حال تعريف مفهوم

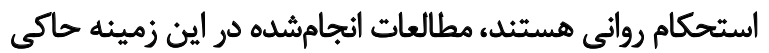

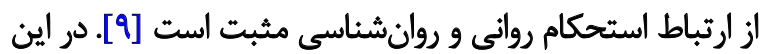

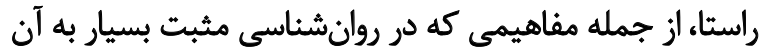

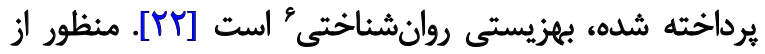

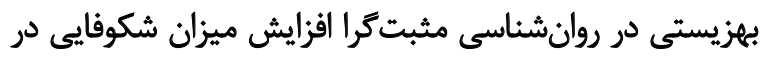

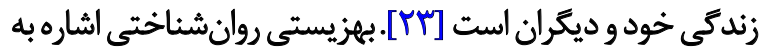

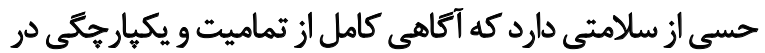

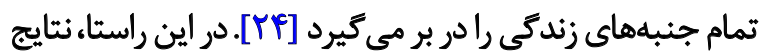

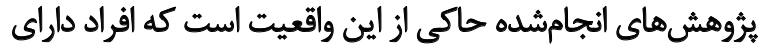

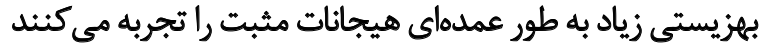

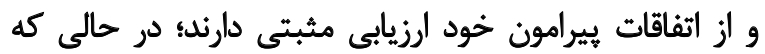

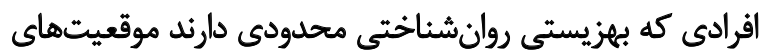

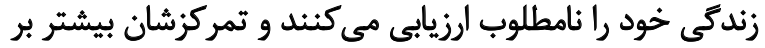

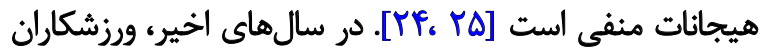

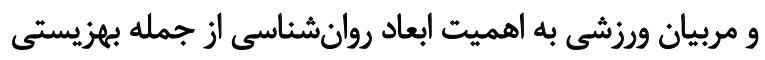

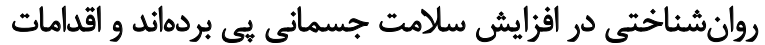

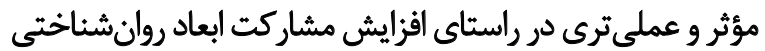




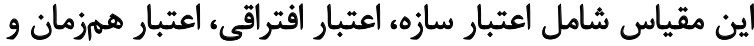

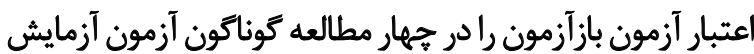

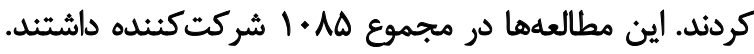

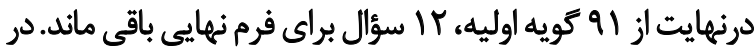

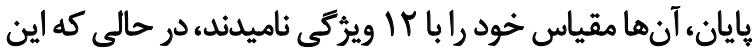

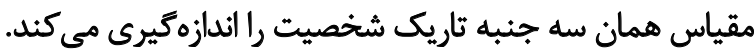

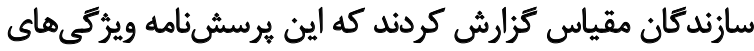

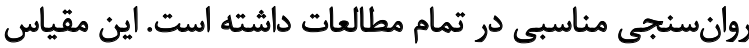

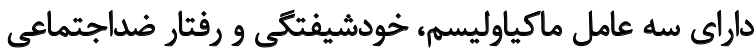

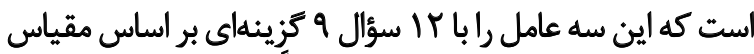

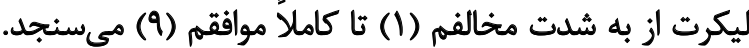

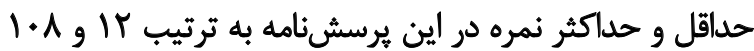

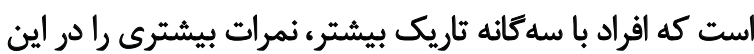

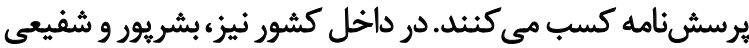

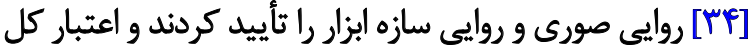

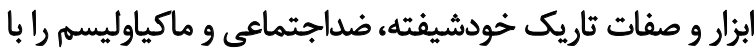

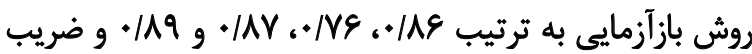

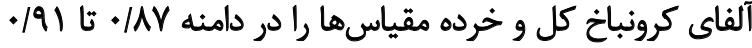

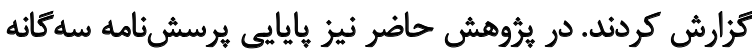

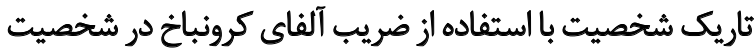

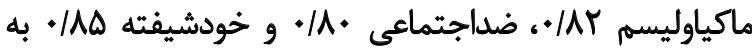

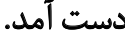

\section{مقياس بهزيستى روان قشئاختي}

اين مقياس را ريف در سال 1919 طراحى كرده است [بrاست.

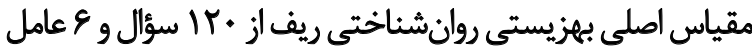

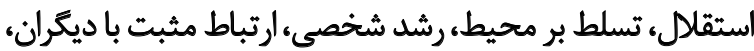

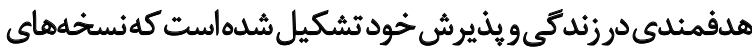

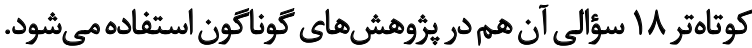

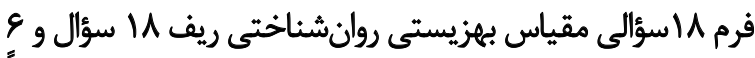

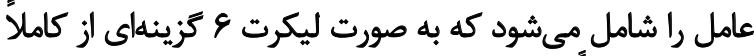

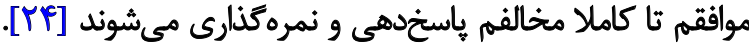

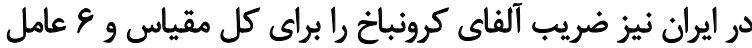

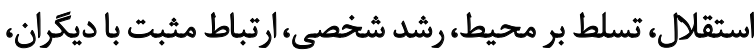

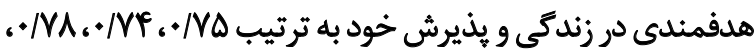

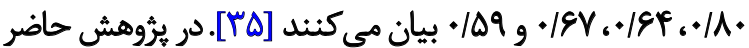

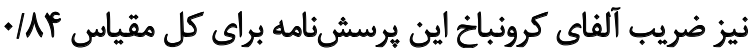

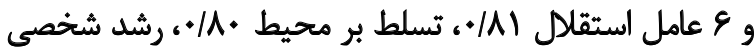

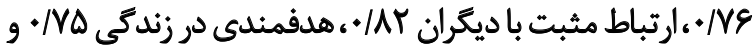

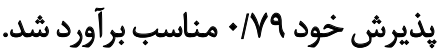

يس إز تكميل يرسشنامهها، دادهها وارد نرمافزار

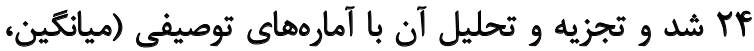

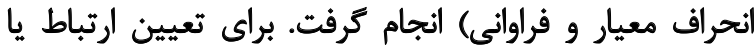

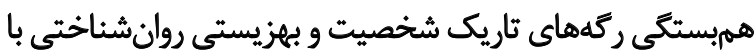

انجام شد؛ كد اخلاق: IR.GUMS.REC.1398.121. سيس

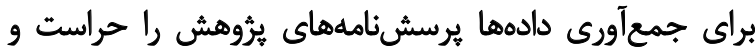

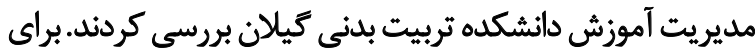

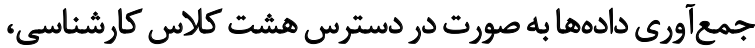

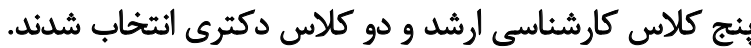

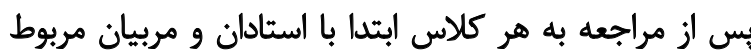

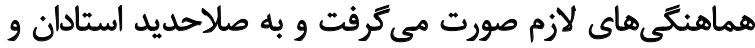

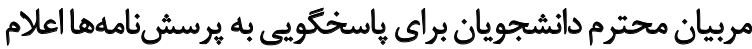

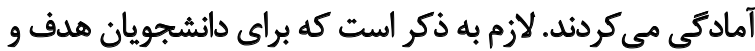

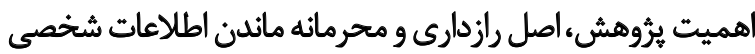

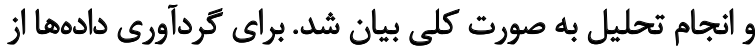

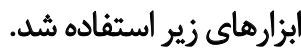

\section{مقياس الستحكام ووالثي ووؤشي}

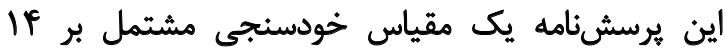

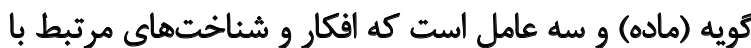

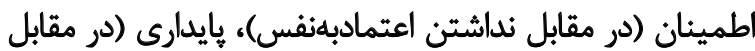

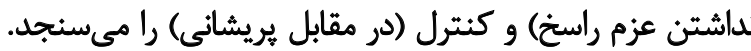

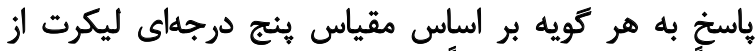

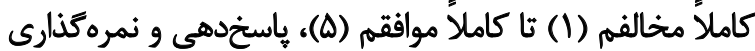

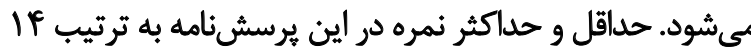

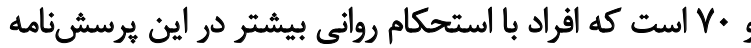

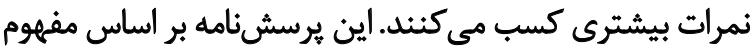

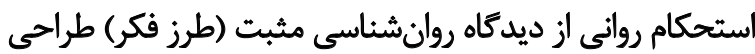

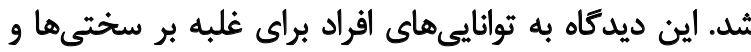

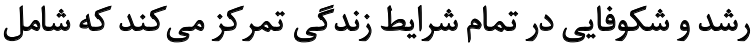

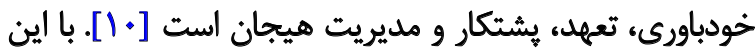

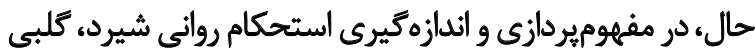

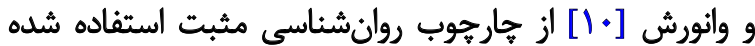

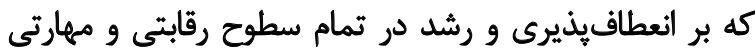

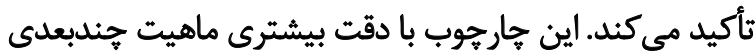

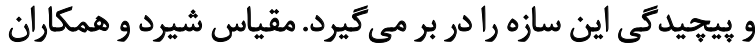

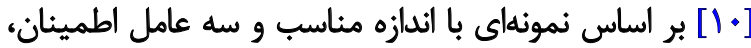

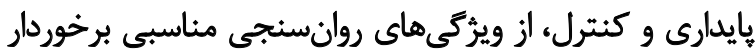

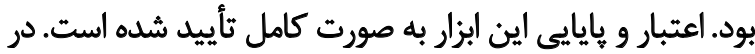

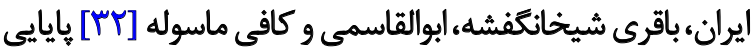

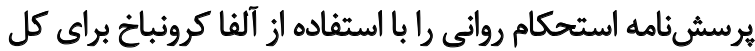

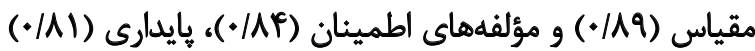

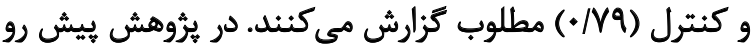

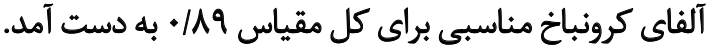

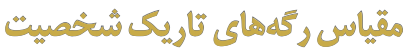

اين مقياس توسط جوناسون ووبستر [Tr]] ساخته شد كه شامل

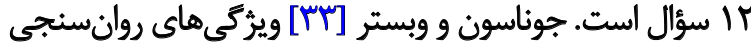


جدول ا. اطلاعات جمعيتشناختى دائشجويان ورزشكار

\begin{tabular}{|c|c|c|}
\hline تعداد (درصد) & \multicolumn{2}{|c|}{ متغيرها } \\
\hline $\operatorname{Pr}(T / H)$ & MEM & \multirow{4}{*}{ دامنه سنى } \\
\hline $9 \pi(f \& / \Delta)$ & "ritr & \\
\hline$\Delta)(r \Delta / \Delta)$ & rq & \\
\hline If $(\mathrm{N} / \mathrm{)}$ & ل كب به بالا & \\
\hline$\Delta(r q \cdot)$ & فوتبال & \multirow{5}{*}{ رشته ورزشى دائشجويان } \\
\hline$\pi(M / \Delta)$ & واليبال & \\
\hline $\operatorname{AV}(\pi / \theta)$ & بل بلنسازي & \\
\hline $\operatorname{IV}(N \Delta)$ & كشتى & \\
\hline $10(V / \Delta)$ & بسكثبال & \\
\hline$I T \Delta(g r / \Delta)$ & كارشئاسى & \multirow{3}{*}{ مقطع تحصيلى } \\
\hline$P V(M / Q)$ & كارشناسىارشد & \\
\hline$r A(\mid \varphi \%)$ & دكترى & \\
\hline $1 T \Delta(\Phi V / \Delta)$ & 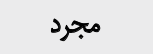 & \multirow{2}{*}{ وضعيت تأهل } \\
\hline$g \Delta(M T / \Delta)$ & متأهل & \\
\hline$M(1 \Delta / \Delta)$ & 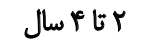 & \multirow{3}{*}{ مدتزمان فعاليت ورزشى } \\
\hline $1.1(\Delta+/ \Delta)$ & ه ه V Vال & \\
\hline \&A $(M \varphi / \cdot)$ & A سال به بالا & \\
\hline
\end{tabular}

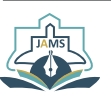

جدول r. شاخصهاى توصيفى و نتايج بررسى نُرمال بودن متغيرهاى يُوهش

\begin{tabular}{|c|c|c|c|}
\hline $\mathbf{P}$ & شاييرو ويلك & ميانكين土|نحراف معيار & هتغيرهاى يرؤوهش \\
\hline .1 .90 & $\cdot 19 \cdot r$ & $\Delta Y / 9 Y \pm V / Q Y$ & استحكام روائى \\
\hline . IFT &.$/ 914$ & $|F / r r \pm g / V|$ & ماكياوليسم \\
\hline.$/ T \Delta V$ & .1941 & $10 / W \pm N \cdot q$ & رفتار ضلفاجتماعي \\
\hline $.1 . \Delta 9$ & - /A9Y & $\mid 8 / 81 \pm E / 81$ & خوششيفتكى \\
\hline.$/ M I A$ &.$/ 917$ & $1 . / r q \pm r / r)$ & استثالال \\
\hline . /MAY &.$/ 918$ & $1 . / 1 \Delta \pm r / \% q$ & تسلط بر هحيط \\
\hline$+1 \odot \Delta Q$ & - /AVD & W/EE $\mathrm{H} / \mathrm{MA}$ & رشد شخصى \\
\hline .1 .84 & . /А9ץ & $1 \cdot / r) \pm r / \Delta A$ & ارتباط مثبت با ديكران \\
\hline.$/ 199$ & $\cdot 19+V$ & $|l / F| \pm r / q \Lambda$ & هدفمثلى در زندكى \\
\hline$+1 \cdot v^{e}$ & INE & $q / r v \pm r / \cdot r$ & يذيرش خود \\
\hline
\end{tabular}


جدول ا. نتايج بروسى همبستكي ركههاي ثاريك شخصيت، بهزيستي روانشئاختي و استحكام روانى

\begin{tabular}{|c|c|c|c|c|c|c|c|c|c|c|c|}
\hline 1. & 9 & $\Lambda$ & $v$ & 7 & $\bullet$ & $\varepsilon$ & $r$ & $r$ & 1 & متغيرها & \\
\hline & & & & & & & & & 1 & ماكياوليسم & 1 \\
\hline & & & & & & & & 1 & . Isarmo & رفتار ضلاجتماعى & r \\
\hline & & & & & & & 1 & $-\cdot / \Delta F \Lambda^{* *}$ & $-.101 \gamma^{* \infty}$ & خوششيفتكى & r \\
\hline & & & & & & 1 & $\cdot(r)+\cdots$ & $-* /$ Tqץ** & $-+|Y A|^{* *}$ & استقلال & r \\
\hline & & & & & 1 & $\Delta / F \Delta \Delta^{* *}$ &.$|F \Delta|^{* *}$ & $-+/ F+\lambda^{* *}$ &.$- / 499 *$ & تسلط بر محيط & $\Delta$ \\
\hline & & & & 1 & . $181 . * *$ & $\cdot / \mu \cdot \Lambda^{* *}$ & . /TqY"ot &.$- / 019 * *$ &.$- / 0 V^{* *}$ & رشد شخصصى & $\varepsilon$ \\
\hline & & & 1 & $.10 \& \Delta^{\circ *}$ &.$|8| Y^{* *}$ & - RAAT** & $\cdot / M \cdot \Delta^{* *}$ & - &.$- / P T \cdot \cdots$ & ارتباط مثبت با ديكران & $\checkmark$ \\
\hline & & 1 & $.(\Delta)+\cdots$ &. $\mid(29)^{-* *}$ & $.18 \cdot 9=0$ & - $/$ fart* & - /roq"* & $-. / r e q=*$ & $-+/ \Delta T+*$ & هدفمندى در زندكى & $\Lambda$ \\
\hline & 1 & -/AYQ** & - $/ \Delta M H=*$ & opien" & . $/ \Delta Y^{*}$ & $\cdot|+1| * *$ & - & $-* / p q \cdot * *$ & $-.18 \Delta 0^{\circ *}$ & يأيرش خود & 9 \\
\hline 1 & $.10 U^{* *}$ &.$/ \Delta \Delta F^{* *}$ & - $10 \rho q * *$ & $.184 \lambda^{* *}$ & . IEPQ" &.$|r \lambda|^{* *}$ & $.1990^{\circ *}$ & $-.1 \Delta W^{* *}$ & $-.18 \cdot p+$ & استحكام روانى & 1. \\
\hline
\end{tabular}

در جلول شُماره Y شاخصهاي توصيفى ركههاي تثاريك شخصيت، بهزيستى روانشناختى و استحكام روانى اعم از ميائكين

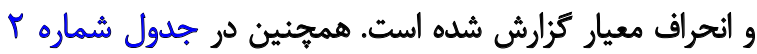

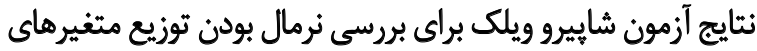

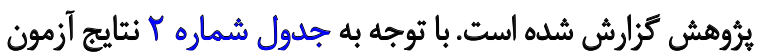

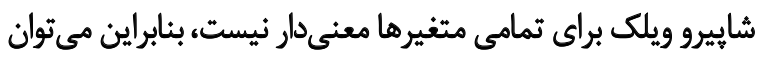
نتيجه كرفت كه توزيع متغيرها نرمال است.

در جدول شماره ب نثايج بررسى همبستىى ركههاى ثاريك
متغير وابسته (استحكام رواني) از ركرسيون جند كائه استفاده شد.

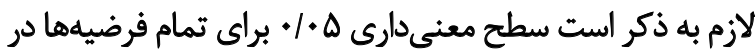
نظر كرفته شد.

Latîn.

اطلاعات جمعيتشناختى دانشجويان ورزشكار از نظر دامنه سنى، رشته ورزشى، مقطع تحصيلى، وضعيت تأهل و مدتزمان جمان

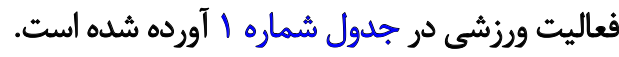

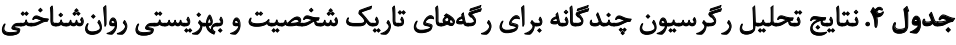

\begin{tabular}{|c|c|c|c|c|c|}
\hline Sig. & $\mathbf{T}$ & $\boldsymbol{\beta}$ & SE & B & متغيرهاى يُوهش \\
\hline $.1 . .1$ & $18 / N A$ & - & $r / I$ & $r \Delta / \Delta$. & مقدار ثابت \\
\hline $.1 \cdot+1$ & $-Y / \& \Delta$ & $-* / 118$ & . $+\Delta T$ &.$- / 1 \mathrm{HA}$ & ماكياوليسم \\
\hline $.1+.1$ & $-r / \Delta q$ &.$- / 1 r 8$ & $.1 \cdot m f$ &.$- / 1 H F$ & رفتار ضداجتماعى \\
\hline $.1++1$ & $r / \Lambda$. &.$/ 118$ & $\cdot 1 \cdot m V$ &.$/ 1 m^{m a}$ & خونشيفتكَى \\
\hline $.1+4$ & $r / .8$ & $.1 . \Delta 9$ & $\cdot 1 \cdot n$ &.$/ 1 P V$ & استقلال \\
\hline.$/ \cdot r A$ & $r / r$ & $1 \cdot 1 \Delta$ & . IrA & - /rat & تسلط بر محيط \\
\hline ( & $r / F q$ & $+1 . q 8$ & .1 .98 &.$/ T F$ & رشد شخصى \\
\hline ( & $r / R F$ & .1 .81 & .1 .41 &.$/ Y I I$ & ارتباط مثبت باديكران \\
\hline$+1+.1$ & $\Delta /+f$ &.$/ N R$ & .1 .91 &.$/ F \Delta q$ & هدفمندى در زندكى \\
\hline $.1 . .1$ & 111. & . TEE & $\cdot / \cdot A r$ & TQM & يذيرش خود \\
\hline
\end{tabular}




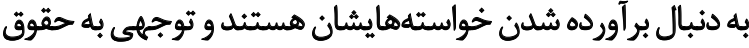

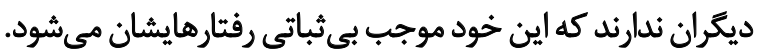

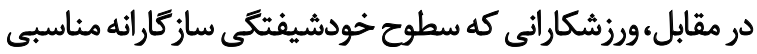

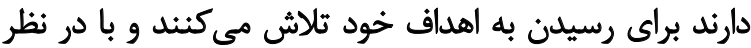

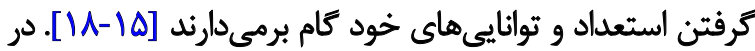

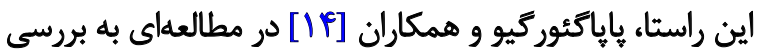

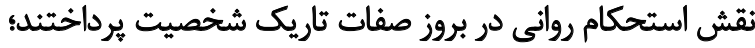

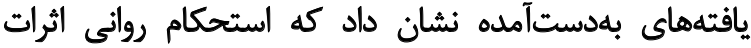

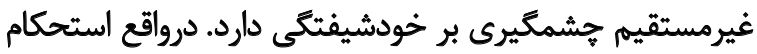

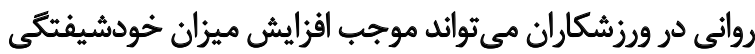

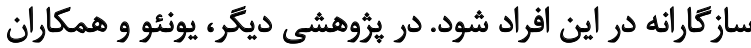

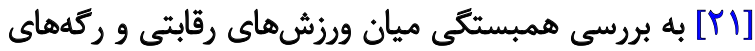

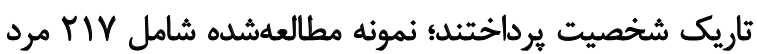

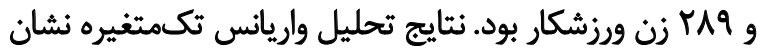

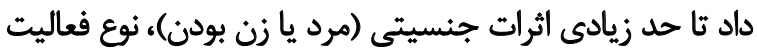

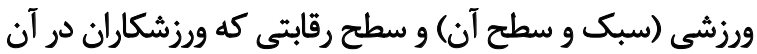

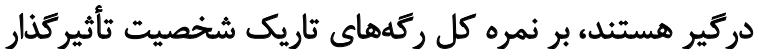

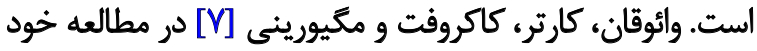

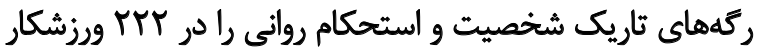

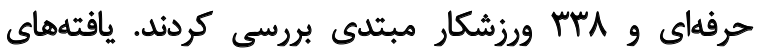

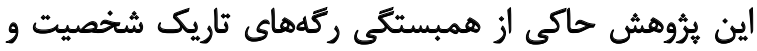

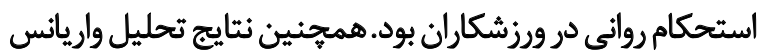

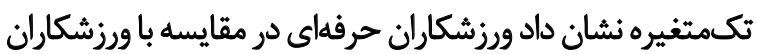

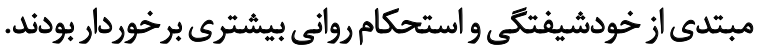

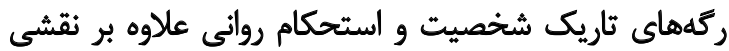

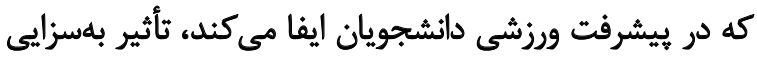

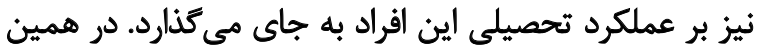

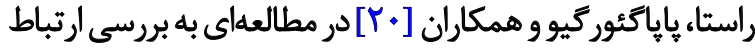
خودشيفتكى، استحكام روانى و موفقيت تحصيلى دانئي دانشجويان

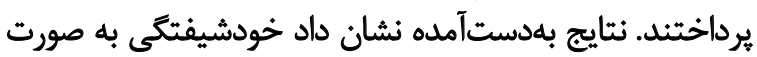

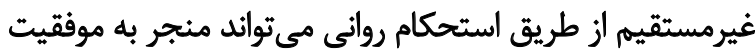

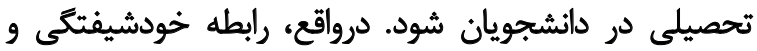

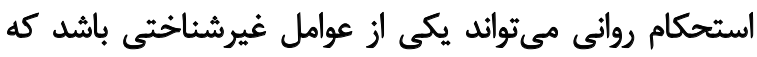

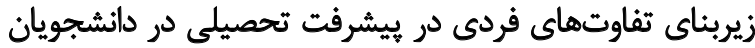

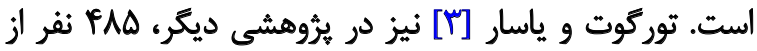

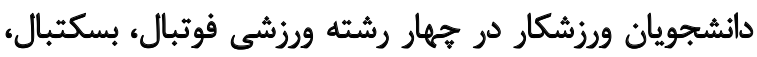

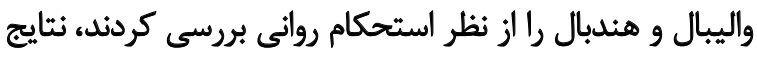

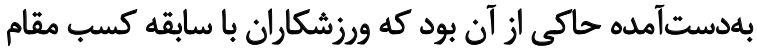

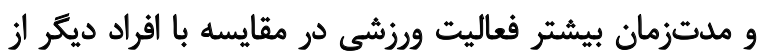
استحكام روانى بيشترى برخوردار بودند.

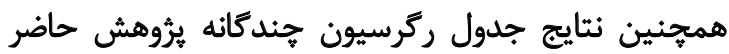

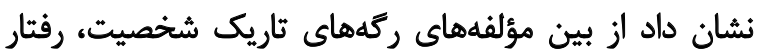

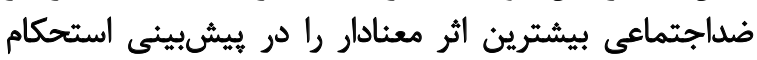

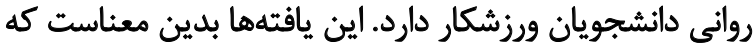

شخصيت، بهزيستى روانشناختى و استحكام روانى دانشجويان

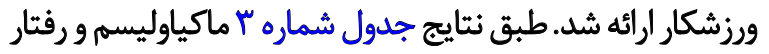

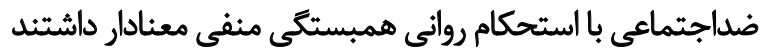

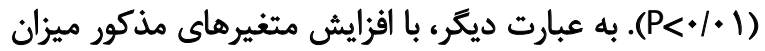

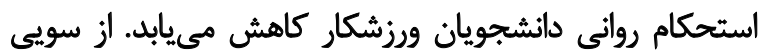

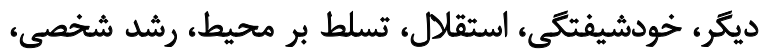

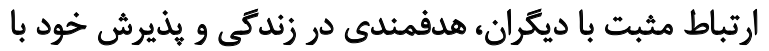

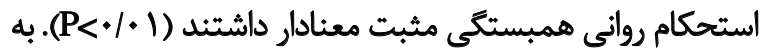

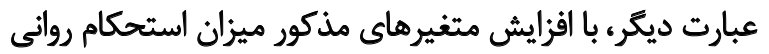
در دانشجويان ورزشكار افزايش مي ميابد.

در جدول شماره f نتايج بررسى نقش رگهدهاى تاريك

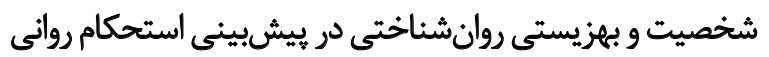

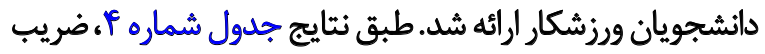

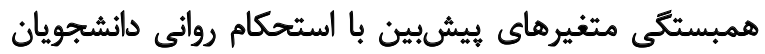

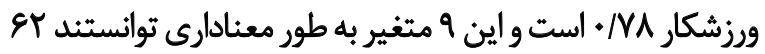

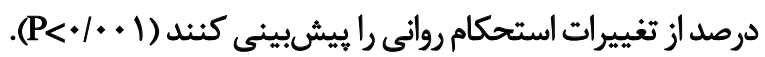

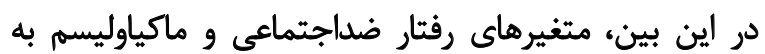

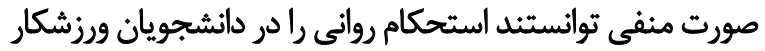

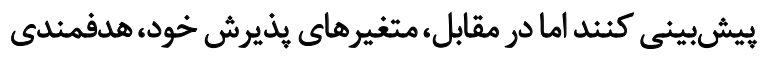

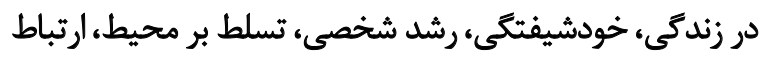

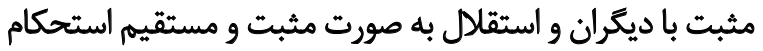

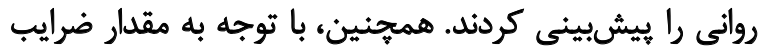

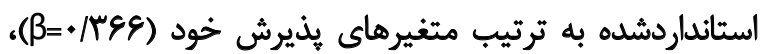

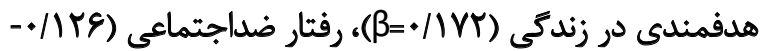

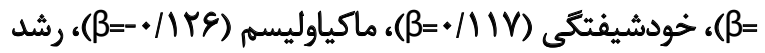

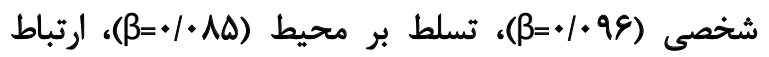

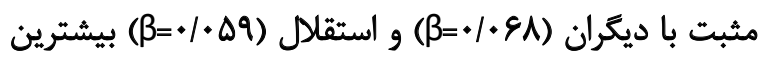
اثر معنادار را در بيشبينى استحكام روان انمانى دانشجويان ورزشكار

داشتيند (ه (A)

ث

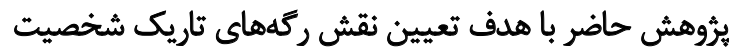

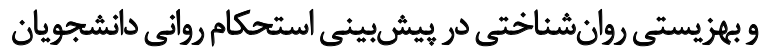

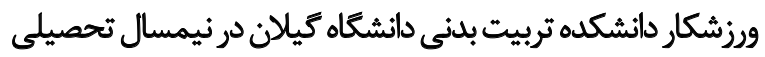

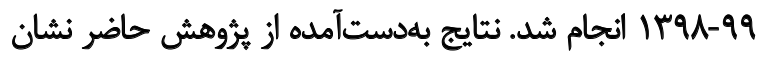

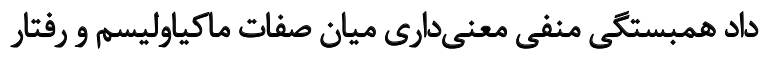

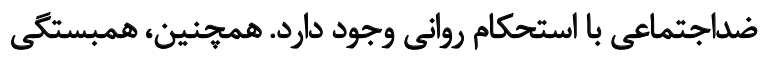

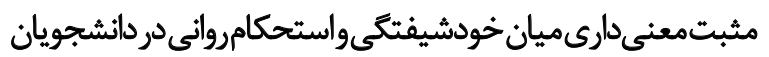

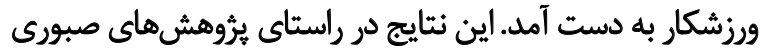

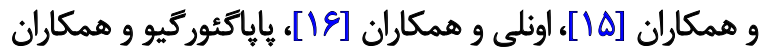

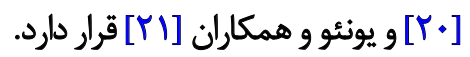
در تبيين اين يافتهها مي توان كفت افرادى كه نمرات بيشترى

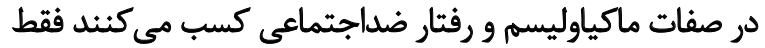




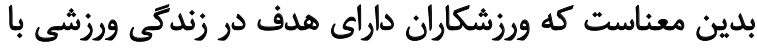

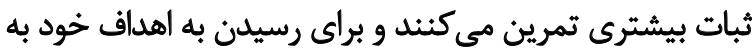

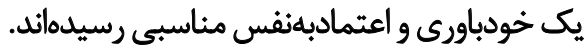

يرؤشش حاضر داراى محدوديتهايي نيز بود؛ براي مثال به داليل

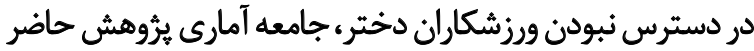

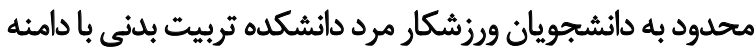

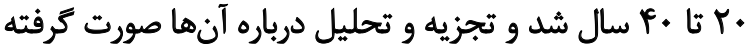

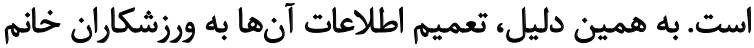

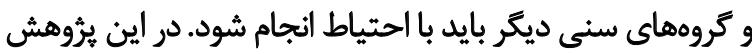

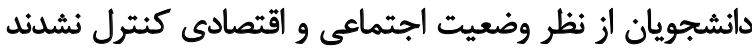

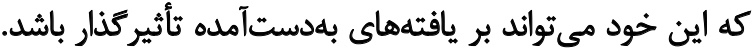

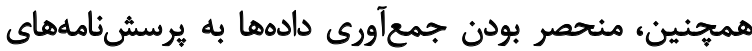

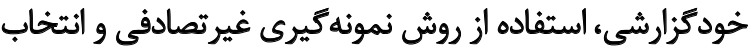

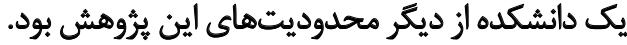
با توجه به اينكه تغذيه و مكملهايى كله ورزشكاران استفاده

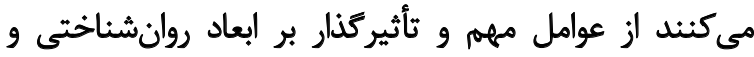

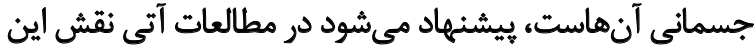

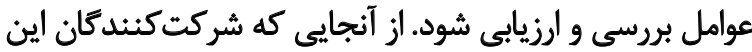

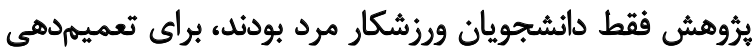

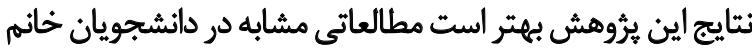

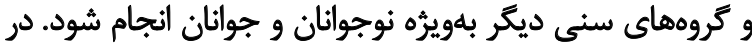

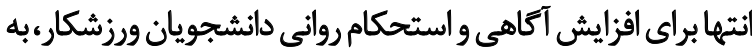

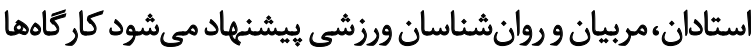
و نشستهايي در راستاى اين موضوع بركّزار كنيند.

\section{تتيجليَّيرى}

در مجموع، نتايج اين يثوهش نشان داد بين صفات ماكياوليسم

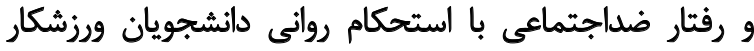

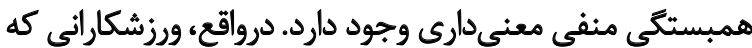

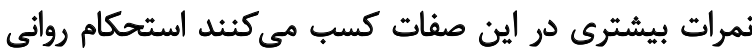

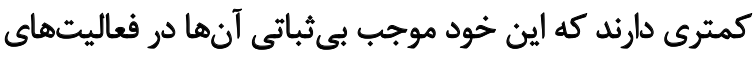

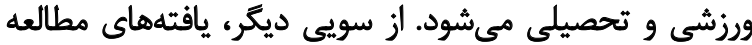

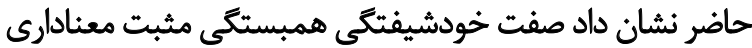

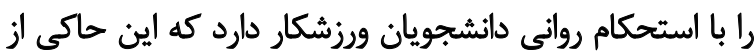

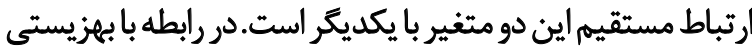

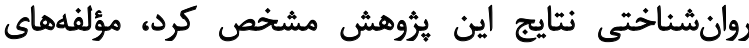

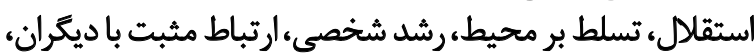

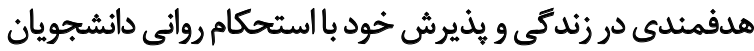

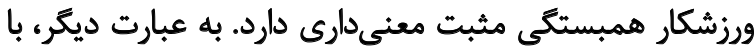

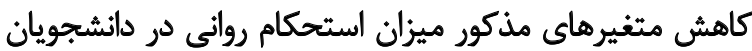

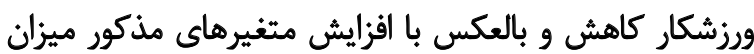

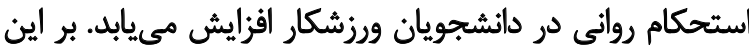

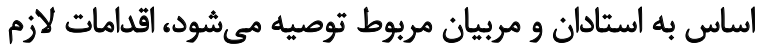

ورزشكارانى با رفتار جامعهستيزائه بيشتر به دنبال كسب قدرت

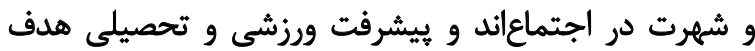

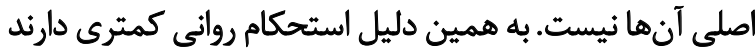

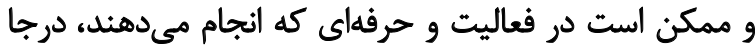

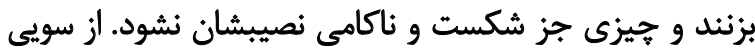

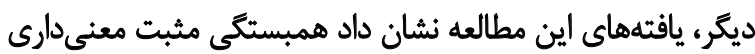

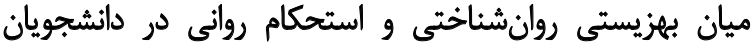
ورزشكار وجود دارد. اين يافتهها همسو با مطالعات ميكو إنولارى

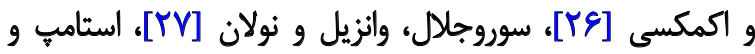

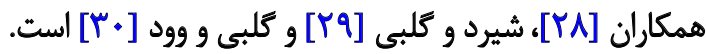
در تبيين اين يافتهها مىتوان اذعان كرد ورزشكارانى كه

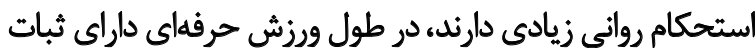

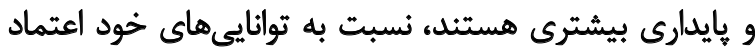

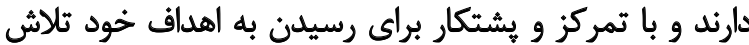

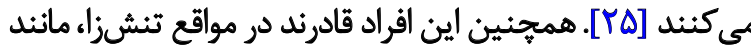

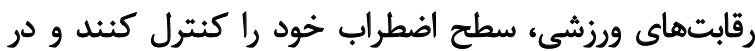

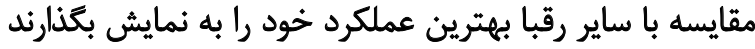

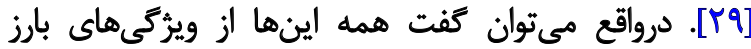

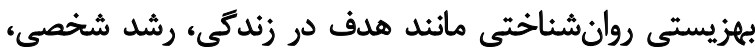

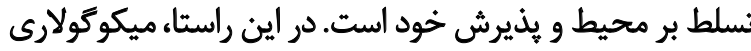

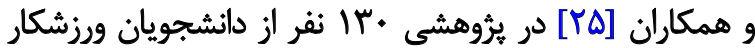

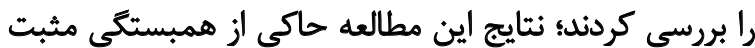

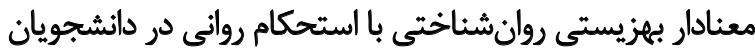

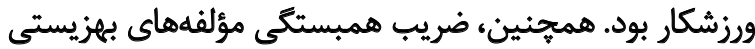

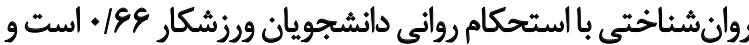

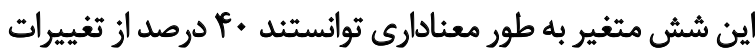

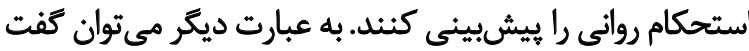

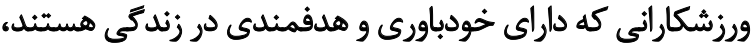

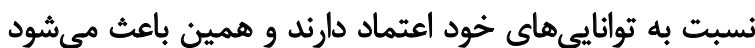

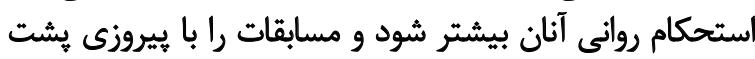

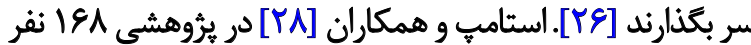

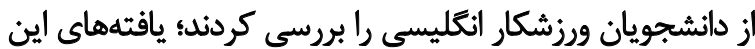

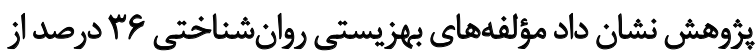

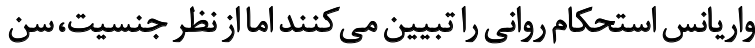

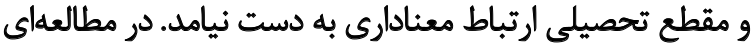

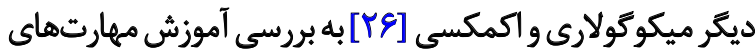

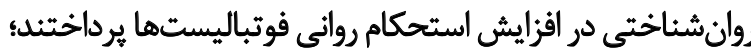

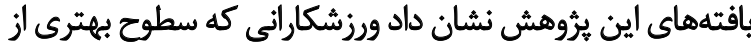

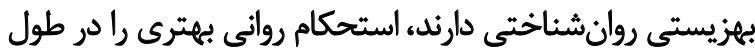
مسابقات از خود به نمايش مي كئنارند.

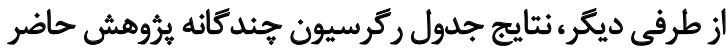

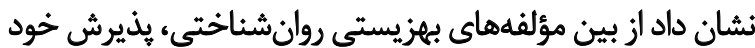

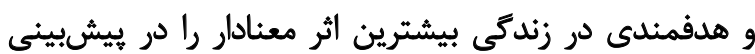
استحكام روانى دانشجويان ورزشكار داشته است. اين يافتهها 
براى بالا بردن بهزيستى روانشناختى دانشجويان رادر دستور كار

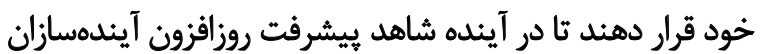
ورزش كشور عزيزمان ايران باشيم.

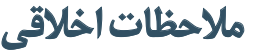

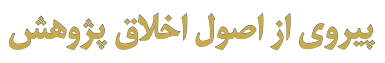
اين مقاله مورد تاييد كميته اخلاق داتشگاه كيلان در

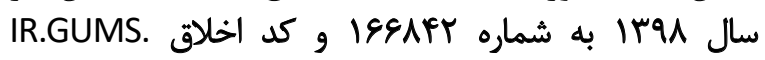
REC.1398.121 قرار كرفته است. Rماره

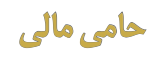

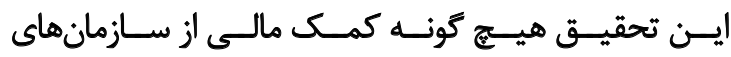

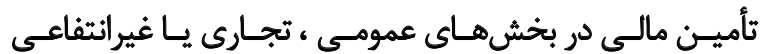

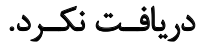

\section{مشار كت نويسند كان}

تمامى نويسندكان در آمادهسازى اين مقاله مشاركت داشتهاند

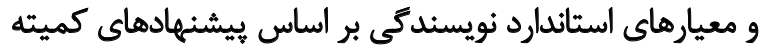
بينالملى ناشران مجلات يزشكى (ICMJE) را داشتند.

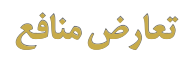

بنابر اظهار نويسندكان اين مقاله تعارض منافع ندارد. 


\section{Refrences}

[1] Stambulova NB, Engström C, Franck A, Linnér L, Lindahl K. Searching for an optimal balance: Dual career experiences of Swedish adolescent athletes. Psychol Sport Exerc. 2015; 21:4-14. [DOI:10.1016/j.psychsport.2014.08.009]

[2] Bowman TG, Pitney WA, Mazerolle SM, Dodge TM. Program directors' perceptions of reasons professional master's athletic training students persist and depart. Athl Train Educ J. 2015; 10(1):57-64. [DOI:10.4085/100157]

[3] Turgut M, Yasar OM. Mental training of college student elite athletes. J Educ Learn. 2020; 9(1):51-9. [DOI:10.5539/jel.v9n1p51]

[4] Cosh S, Tully PJ. Stressors, coping, and support mechanisms for student athletes combining elite sport and tertiary education: Implications for practice. J Sport Psychol. 2015; 29(2):120-33. [DOI:10.1123/ tsp.2014-0102

[5] Van Zyl Y, Surujlal J, Singh C. An empirical study of university student-athlete's strategies for coping with stress: Social psychology of sport. Afr J Physical Health Educ Recreation Dance. 2009; 9(1):62-78. [DOI:10.4314/ ajpherd.v15i4.47282]

[6] Mazerolle SM, Bowman TG, Dodge TM. Athletic training student socialization part I: Socializing students in undergraduate athletic training programs. Athl Train Educ J. 2014; 9(2):72-9. [DOI:10.4085/090272]

[7] Vaughan R, Carter GL, Cockroft D, Maggiorini L. Harder, better, faster, stronger? Mental toughness, the dark triad and physical activity. Pers Individ Differ. 2018; 131:206-11. [DOI:10.1016/j.paid.2018.05.002]

[8] Erdoğan N, Kocaekși S. Psychological characteristic of the elite athletes have required. Turk Klin J Sports Sci. 2015; 7(2):57-64. [DOI:10.5336/ sportsci.2014-42993]

[9] Liew G, Kuan G, Chin N, Hairul AH. Mental toughness in sport: Systematic review and future. Ger J Exerc Sport Res. 2019; 49(4):381-94. [DOI:10.1007/s12662-019-00603-3]

[10] Sheard M, Golby J, Van Wersch A. Progress toward construct validation of the Sports Mental Toughness Questionnaire (SMTQ). Eur J Psychol Assess. 2009; 25(3):186-93. [DOI:10.1027/1015-5759.25.3.186]

[11] Nicholls AR, Morley D, Perry JL. Mentally tough athletes are more aware of unsupportive coaching behaviours: Perceptions of coach behaviour, motivational climate, and mental toughness in sport. Int J Sports Sci Coach. 2016; 11(2):172-81. [DOI:10.1177\%2F1747954116636714]

[12] Gucciardi DF, Hanton S, Gordon S, Mallett CJ, Temby P. The concept of mental toughness: Tests of dimensionality, nomological network, and traitness. J Pers. 2015; 83(1):26-44. [DOI:10.1111/jopy.12079][PMID]

[13] Clough PJ, Earle K, Sewell D. Mental toughness: The concept and its measurement. In: Cockerill I, editor. Solutions in Sport Psychology. London: Thomson. p. 32-43. https://www.scirp.org/ (S(czeh2tfqyw2orz553k1w0r45))/reference/ReferencesPapers. aspx?ReferencelD=1902775

[14] Papageorgiou KA, Wong B, Clough PJ. Beyond good and evil: Exploring the mediating role of mental toughness on the Dark Triad of personality traits. Pers Individ Dif. 2017; 119:19-23. [DOI:10.1016/j. paid.2017.06.031]

[15] Sabouri S, Gerber M, Sadeghi Bahmani S, Lemola S, Clough PJ, Kalak $\mathrm{N}$, et al. Examining dark triad traits in relation to mental toughness and physical activity in young adults. Neuropsychiatr Dis Treat. 2016; 12:229-35. [DOI:10.2147/NDT.S97267][PMID][PMCID]
[16] Onley M, Veselka L, Schermer JA, Vernon PA. Survival of the scheming: A genetically informed link between the dark triad and mental toughness. Twin Res Hum Genet. 2013; 16(6):1087-95. [DOI:10.1017/ thg.2013.66][PMID]

[17] Paulhus DL, Williams KM. The dark triad of personality: Narcissism, Machiavellianism, and psychopathy. J Res Pers. 2002; 36(6):556-663. [DOI:10.1016/S0092-6566(02)00505-6]

[18] Rauthmann JF, Kolar GP. How "dark" are the Dark Triad traits? Examining the perceived darkness of narcissism, Machiavellianism, and psychopathy. J Pers Individ Differ. 2012; 53(7):884-9. [DOI:10.1016/j. paid.2012.06.020]

[19] Tazegül Ü. Comparison of the narcissism level of the bodybuilders before and after the application of anabolic steroid cure. Univers J Educ Res. 2018; 6(6):1149-52. [DOI:10.13189/ujer.2018.060603]

[20] Papageorgiou KA, Malanchini M, Denovan A, Clough PJ, Shakeshaft N Schofield K, et al. Longitudinal associations between narcissism, mental toughness and school achievement. Pers Individ Differ. 2018; 131:10510. [DOI:10.1016/j.paid.2018.04.024]

[21] Ueno Y, Shimotsukasa T, Suyama S, Oshio A. Correlations between competitive sports' characteristics and the dark triad. J Phys Educ Sport. 2017; 17(2):533-6. [DOI:10.7752/jpes.2017.02080]

[22] Lambert L, Passmore HA, Holder MD. Foundational frameworks of positive psychology: Mapping well-being orientations. Can Psychol. 2015; 56(3):311-21. [DOI:10.1037/cap0000033]

[23] Ryff CD. Happiness is everything, or is it? Explorations on the mean ing of psychological well-being. J Pers Soc Psychol. 1989; 57(6):1069-81. [DOI:10.1037/0022-3514.57.6.1069]

[24] Ryff CD. Psychological well-being revisited: Advances in the science and practice of eudaimonia. Psychother Psychosom. 2014; 83(1):10-28. [DOI:10.1159/000353263] [PMID] [PMCID]

[25] Micoogullari BO, Odek U, Beyaz O. Evaluation of sport mental toughness and psychological wellbeing in undergraduate student athletes. Educ Res Rev. 2017; 12(8):483-7. [DOI:10.5897/ERR2017.3216]

[26] Miçoogullari BO, Ekmekçi R. Evaluation of a psychological skill training program on mental toughness and psychological wellbeing for professional soccer players. Univers J Educ Res. 2017; 5(12):2312-9. [DOI:10.13189/ujer.2017.051222]

[27] Surujlal J, Van Zyl Y, Nolan VT. Perceived stress and coping skills of university student-athletes and the relationship with life satisfaction. Afr J Phys Health Educ, Recreat Dance. 2013; 19(42):1047-59. https://hdl. handle.net/10520/EJC145373

[28] Stamp E, Crust L, Swann C, Perry J, Clough P, Marchant D. Relationships between mental toughness and psychological wellbeing in undergraduate students. Pers Individ Differ. 2015; 75:170-4. [DOI:10.1016/j. paid.2014.11.038]

[29] Sheard M, Golby J. Effect of a psychological skills training program on swimming performance and positive psychological development. Int J Sport Exerc Psychol. 2006; 4(2):149-69. [DOI:10.1080/161219 7X.2006.9671790]

[30] Golby J, Wood P. The effects of psychological skills training on mental toughness and psychological well-being of student-athletes. J Psychol. 2016; 7(06):901-3. [DOI:10.4236/psych.2016.76092]

[31] Etikan I, Musa SA, Alkassim RS. Comparison of convenience sampling and purposive sampling. Am J Theor Appl Stat. 2016; 5(1):1-4 [DOI:10.11648/j.ajtas.20160501.11] 
[32] Bagheri Sheykhangafshe F, Abolghasemi A, Kafi Masouleh S. [Comparison dark triad traits of personality, cognitive decision-making and mental toughness in natural, supplement and steroid bodybuilders (Persian)]. Sport Psy Stud. 2019; 8(29):19-38. [DOI:10.22089/ spsyj.2019.7931.1854]

[33] Jonason PK, Webster GD. The dirty dozen: A concise measure of the dark triad. J Psychol Assess. 2010; 22(2):420-32. [DOI:10.1037/ a0019265]

[34] Basharpoor S, Shafie M. [Psychometric properties of Persian version of short form scale of dark triad personality traits in university students (Persian)]. J Res Psychol Health. 2015; 9(1):1. https://rph.khu.ac.ir/article-1-2459-en.html

[35] Bagheri Sheykhangafshe F, Shabahang R. [Prediction of psychological wellbeing of elderly people based on spirituality, social support, and optimism (Persian)]. J Religion Health. 2020; 7(2):22-32. http://jrh.mazums.ac.ir/article-1-716-en.html 